This paper is a postprint of a paper submitted to and accepted for publication in

IET Communications (Special issue on Cognitive Communications) and is subject to

Institution of Engineering and Technology Copyright.

The copy of record is available at IET Digital Library.

\title{
Improved Energy Detection Spectrum Sensing for Cognitive Radio
}

Miguel López-Benítez and Fernando Casadevall

Department of Signal Theory and Communications

Universitat Politècnica de Catalunya, Barcelona, Spain

E-mail: \{miguel.lopez, ferranc\}etsc.upc.edu

\begin{abstract}
Energy detection constitutes a preferred approach for spectrum sensing in cognitive radio due to its simplicity and applicability (it works irrespective of the signal format to be detected) as well as its low computational and implementation costs. The main drawback, however, is its well-known detection performance limitations. Various alternative detection methods have been shown to outperform energy detection, but at the expense of increased complexity and confined field of applicability. In this context, this work proposes and evaluates an improved version of the energy detection algorithm that is able to outperform the classical energy detection scheme while preserving a similar level of algorithm complexity as well as its general applicability regardless of the particular signal format or structure to be detected. The performance improvement is evaluated analytically and corroborated with experimental results.
\end{abstract}

Index terms - Cognitive radio; dynamic spectrum access; spectrum sensing; energy detection.

\section{INTRODUCTION}

While still early in its development, Cognitive Radio (CR) [1, 2] has emerged as a promising solution that can effectively address the existing conflicts between spectrum demand growth and spectrum underutilization. CR aims at improving spectrum usage efficiency by allowing some unlicensed (secondary) users to access in an opportunistic and non-interfering manner some licensed bands temporarily unoccupied by the licensed (primary) users.

One of the most important challenges for a CR network is not to cause harmful interference to primary users. To guarantee interference-free spectrum access, secondary users should reliably 
identify the presence of primary users, which basically means being able to determine whether a primary signal is present within a certain frequency range [3]. A number of different signal detection methods, referred to as spectrum sensing algorithms in the context of CR, have been proposed in the literature to identify the presence of primary signal transmissions [4-6]. Some examples of the existing proposals include energy detection [7], matched filter detection [8, 9], cyclostationary feature detection [10,11], covariance-based detection [12], multi-taper spectrum estimation [13] and filter bank spectrum estimation [14]. The existing solutions provide different trade-offs between required sensing time, complexity and detection capabilities, but their practical applicability depends on how much information is available about the primary user signal. In the most generic case, a CR user is not expected to be provided with any prior information about the primary signals that may be present within a certain frequency band. When the secondary receiver cannot gather sufficient information, the energy detection principle [7] can be used due to its ability to work irrespective of the signal structure to be detected. Despite its practical performance limitations, energy detection has gained popularity as a spectrum sensing technique for CR due to its general applicability and simplicity as well as its low computational and implementation costs. Energy detection has been a preferred approach for many past spectrum sensing studies and also constitutes the spectrum sensing method studied in this work.

Energy detection compares the signal energy received in a certain frequency band to a properly set decision threshold. If the signal energy lies above the threshold, the band is declared to be busy. Otherwise the band is supposed to be idle and could be accessed by CR users. Due to the generality of its operating principle, the performance of energy detection would not be expected to depend on the type of primary signal being detected. However, a recent study [15] based on empirical measurements of various real-world signals demonstrated that the detection performance of energy detection may strongly vary with the primary radio technology being detected. Certain technologydependent inherent properties may result in different detection performances for various primary signals under the same operating conditions. In other words, the resulting probability of detection 
for a fixed set of operating parameters might be enough to reliably detect some primary signals but might not for some others, thus making some radio technologies more susceptible to interferences under the same operating conditions. A possibility to overcome this drawback would be to employ other more sophisticated spectrum sensing algorithms, which have been proven to outperform the classical energy detection scheme. Such methods, however, have usually been devised for the improved detection of particular signal formats, which restricts their field of applicability to a few primary radio technologies. Furthermore, the performance improvement provided by such methods is normally obtained at the expense of significantly increased algorithm complexity and computational cost. An ideal spectrum sensing algorithm should be able to provide detection performance improvements for any primary signal without incurring in excessive implementation and computational costs. In this context, this work proposes and evaluates, both analytically and experimentally, an improved version of the energy detection algorithm. The main interest of the proposed scheme relies on its ability to outperform the classical energy detection scheme while preserving a similar level of algorithm complexity as well as its general applicability regardless of the particular signal format or structure to be detected. The performance improvement is studied analytically and corroborated with experimental results.

\section{SPECTRUM SENSING PROBLEM FORMULATION}

The spectrum sensing problem can be formulated as a binary hypothesis testing problem with the following two hypotheses:

$$
\begin{array}{ll}
\mathcal{H}_{0}: y[n]=w[n] & n=1,2, \ldots, N \\
\mathcal{H}_{1}: y[n]=x[n]+w[n] & n=1,2, \ldots, N
\end{array}
$$

where $\mathcal{H}_{0}$ is a null hypothesis stating that the received signal samples $y[n]$ correspond to noise samples $w[n]$ and therefore there is no primary signal in the sensed spectrum band, and hypothesis $\mathcal{H}_{1}$ indicates that some licensed user signal $x[n]$ is present. $N$ denotes the number of samples collected during the signal observation interval (i.e., the sensing period), emphasizing that the de- 
cision is made based on a limited number of signal samples. The ideal spectrum sensor would select hypothesis $\mathcal{H}_{1}$ whenever a primary signal is present and hypothesis $\mathcal{H}_{0}$ otherwise. Unfortunately, spectrum sensing algorithms may fall into mistakes in practice, which can be classified into missed detections and false alarms. A missed detection occurs when a primary signal is present in the sensed band and the spectrum sensing algorithm selects hypothesis $\mathcal{H}_{0}$, which may result in harmful interference to primary users. On the other hand, a false alarm occurs when the sensed spectrum band is idle and the spectrum sensing algorithm selects hypothesis $\mathcal{H}_{1}$, which results in missed transmission opportunities and therefore in a lower spectrum utilization. Based on these definitions, the performance of any spectrum sensing algorithm can be summarized by means of two probabilities: the probability of missed detection $P_{m d}=P\left(\mathcal{H}_{0} / \mathcal{H}_{1}\right)$, or its complementary probability of detection $P_{d}=P\left(\mathcal{H}_{1} / \mathcal{H}_{1}\right)=1-P_{m d}$, and the probability of false alarm $P_{f a}=P\left(\mathcal{H}_{1} / \mathcal{H}_{0}\right)$. Large $P_{d}$ and low $P_{f a}$ values would be desirable. Nevertheless, there exists a trade-off between $P_{d}$ and $P_{f a}$, meaning that improving one of these performance metrics in general implies degrading the other one and vice versa. In this context, Receiver Operating Characteristic (ROC) curves (obtained by plotting $P_{d}$ versus $P_{f a}$ ) are very useful since they allow us to explore the relationship between the sensitivity $\left(P_{d}\right)$ and specificity $\left(P_{f a}\right)$ of a spectrum sensing method for a variety of different algorithm parameters and other affecting factors.

\section{Classical energy detection}

\section{III.A. Operating principle}

The Classical Energy Detection (CED) principle (see algorithm 1), also referred to as radiometric detection, measures the energy received on a primary band during an observation interval and declares the current channel state $S_{i}$ as busy (hypothesis $\mathcal{H}_{1}$ ) if the measured energy is greater than a properly set predefined threshold, or idle (hypothesis $\mathcal{H}_{0}$ ) otherwise [7]:

$$
\mathbb{T}_{i}\left(\mathbf{y}_{i}\right)=\sum_{n=1}^{N}\left|y_{i}[n]\right|^{2} \underset{\mathcal{H}_{0}}{\stackrel{\mathcal{H}_{1}}{\gtrless}} \lambda
$$


where $\mathbb{T}_{i}\left(\mathbf{y}_{i}\right)$ is the test statistic computed in the $i$-th sensing event over the signal vector $\mathbf{y}_{i}=$ $\left(y_{i}[1], y_{i}[2], \ldots, y_{i}[N]\right)$, and $\lambda$ is a fixed decision threshold to distinguish between the two hypotheses in equation 1.

\section{III.B. Theoretical performance}

Closed-form expressions for the detection $\left(P_{d}\right)$ and false alarm $\left(P_{f a}\right)$ probabilities can be obtained based on the statistics of $\mathbb{T}_{i}\left(\mathbf{y}_{i}\right)$. The test statistic follows a central (under hypothesis $\mathcal{H}_{0}$ ) and non-central (under hypothesis $\mathcal{H}_{1}$ ) chi-square distribution with $2 N$ degrees of freedom [7]. Notice, however, that the non-interference constraint for CR terminals imposes strict detection performance requirements that must be met even in the worst possible operating case, i.e. low Signal-to-Noise Ratio (SNR) conditions ${ }^{1}$. In low SNR regimes, the number of signal samples required to achieve a certain performance is usually large $(N \gg 1)$. Based on this observation, the central limit theorem can therefore be employed to approximate the test statistic as Gaussian:

$$
\mathbb{T}_{i}\left(\mathbf{y}_{i}\right) \sim \begin{cases}\mathcal{N}\left(N \sigma_{w}^{2}, 2 N \sigma_{w}^{4}\right), & \mathcal{H}_{0} \\ \mathcal{N}\left(N\left(\sigma_{x}^{2}+\sigma_{w}^{2}\right), 2 N\left(\sigma_{x}^{2}+\sigma_{w}^{2}\right)^{2}\right), & \mathcal{H}_{1}\end{cases}
$$

where $\sigma_{x}^{2}$ is the received average primary signal power and $\sigma_{w}^{2}$ is the noise variance. If only Additive White Gaussian Noise (AWGN) is considered, the $P_{d}$ and $P_{f a}$ for the CED algorithm can then be obtained based on the statistics of $\mathbb{T}_{i}\left(\mathbf{y}_{i}\right)$ as follows:

$$
\begin{aligned}
P_{d}^{\mathrm{CED}} & =P\left\{\mathbb{T}_{i}\left(\mathbf{y}_{i}\right)>\lambda\right\}_{\mathcal{H}_{1}}=\mathcal{Q}\left(\frac{\lambda-N\left(\sigma_{x}^{2}+\sigma_{w}^{2}\right)}{\sqrt{2 N\left(\sigma_{x}^{2}+\sigma_{w}^{2}\right)^{2}}}\right) \\
P_{f a}^{\mathrm{CED}} & =P\left\{\mathbb{T}_{i}\left(\mathbf{y}_{i}\right)>\lambda\right\}_{\mathcal{H}_{0}}=\mathcal{Q}\left(\frac{\lambda-N \sigma_{w}^{2}}{\sqrt{2 N \sigma_{w}^{4}}}\right)
\end{aligned}
$$

where $P\{A\}_{B} \triangleq P(A \mid B)$ and $\mathcal{Q}(\cdot)$ is the Gaussian tail probability $Q$-function [17, (26.2.3)].

\footnotetext{
${ }^{1}$ For example, the IEEE 802.22 spectrum sensing requirements specify that a CR terminal must be able to detect a 6-MHz digital TV signal at a power level of $-116 \mathrm{dBm}$ [16], which corresponds to a SNR of $-21 \mathrm{~dB}$ for a typical TV receiver with a noise figure of $11 \mathrm{~dB}[16]$.
} 


\section{III.C. Threshold setting}

The procedure employed to select the algorithm's decision threshold is an important aspect since it represents the parameter configured by the system designer to control the spectrum sensing performance. The decision threshold $\lambda$ could be chosen for an optimum trade-off between $P_{d}$ and $P_{f a}$. However, this would require knowledge of noise and detected signal powers. While the noise power can be estimated, the signal power is difficult to estimate since it depends on many varying factors such as transmission and propagation characteristics. In practice, the threshold is normally chosen to satisfy a certain $P_{f a}$ [18], which only requires the noise power to be known. Solving in equation 5 for $\lambda$ yields the decision threshold required for a target probability of false alarm:

$$
\lambda=\left(\mathcal{Q}^{-1}\left(P_{f a, \text { target }}^{\text {CED }}\right) \sqrt{2 N}+N\right) \sigma_{w}^{2}
$$

Substituting equation 6 into equation 4 and dividing numerator and denominator by $\sigma_{w}^{2}$ yields:

$$
P_{d}^{\mathrm{CED}}(\gamma)=\mathcal{Q}\left(\frac{\mathcal{Q}^{-1}\left(P_{f a, \text { target }}^{\text {CED }}\right) \sqrt{2 N}-N \gamma}{\sqrt{2 N}(1+\gamma)}\right) \approx \mathcal{Q}\left(\mathcal{Q}^{-1}\left(P_{f a, \text { target }}^{\mathrm{CED}}\right)-\sqrt{\frac{N}{2} \gamma}\right)
$$

which represents the detection probability of the CED algorithm as a function of the SNR, denoted as $\gamma=\sigma_{x}^{2} / \sigma_{w}^{2}$. The approximation of the last term assumes the low SNR regime case $(\gamma \ll 1)$.

\section{III.D. Experimental performance}

Figure 1 shows the theoretical and experimental performance of the CED method when applied to real-world primary signals of various radio technologies, including analogical and digital TV, DAB-T, TETRA, E-GSM 900, DCS 1800 and UMTS. A detailed and in-depth description of the measurement platform as well as the measurement and evaluation methodologies employed to obtain these results can be found in [15].

There are two important aspects to be mentioned in Figure 1. First, the experimental results indicate that the detection performance may notably vary with the radio technology being detected, which is not predicted by the associated theoretical results (see equation 7). As a matter of fact, for 
a given set of operating parameters (target $P_{f a}$, sample length $N$ and SNR $\gamma$ ) equation 7 suggests that the resulting performance in terms of $P_{d}$ is unique. However, Figure 1 clearly demonstrates that the experimental $P_{d}$ may strongly depend on the primary signal being sensed. Second, the performance differences among various radio technologies are not constant, but depend on the sensing period $N$. Summarizing the analysis and discussion of [15], this behavior can be explained as follows. If $N$ is sufficiently low, the test statistic may follow the instantaneous variations of the received signal energy. Under the same average SNR conditions (i.e., signals with the same average energy, assuming constant average noise energy), this means that a higher signal energy variability (variance) implies a higher probability that the instantaneous energy level (and the test statistic) falls below the decision threshold. In such a case, the channel would be declared as idle even if it should be declared as busy, thus resulting in a degraded detection performance. Since various radio technologies may exhibit different signal energy variation patterns and variances, this explains the different detection performances observed in Figure 1. It is interesting to observe that sufficiently short sensing periods $(N=10)$ are not able to provide reliable estimates of the signal energy. The test statistic values obtained for $N=10$ are highly variable and in an important number of cases they fall below the decision threshold even in the presence of a primary signal. This leads to an important number of signal misdetections and thus to a significant detection performance degradation with respect to the theoretical prediction of equation 7. As $N$ increases, the test statistics are computed over longer observation periods, thus averaging the peculiarities of any instantaneous energy variation pattern and reducing its variance. In such a case, although the variability of the received energy remains the same, the variability of the test statistic decreases and so does the probability of misdetecting the primary signal. For sufficiently long observation periods, the test statistic ceases to follow the instantaneous signal energy variations and its value closely resembles the true mean signal energy. When this occurs for all the considered signals, the obtained performance curves converge. This explains the convergent trend observed in Figure 1 as $N$ increases. 


\section{IMPROVED ENERGY DETECTION}

To overcome the limitations of the CED scheme, a novel energy detection-based spectrum sensing method is proposed. For the shake of clarity, the proposal is presented in two steps. Firstly, a Modified Energy Detection (MED) method motivated by the experimental results of Section III.D. is presented. Afterwards, and based on the MED scheme, a refined version, referred to as Improved Energy Detection (IED) method, is proposed and analyzed.

\section{IV.A. MED operating principle}

The experimental performance observed in Section III.D. for the CED scheme suggests that the detection performance might be improved if the misdetections caused by instantaneous signal energy drops could be avoided. This motivates the development of the MED scheme (see algorithm 2). Every sensing event, the MED method computes the test statistic $\mathbb{T}_{i}\left(\mathbf{y}_{i}\right)$ as performed by the CED method (see equation 2). The main difference between the MED and the CED algorithms is that the former additionally maintains an updated list containing the test statistic values of the last $L$ sensing events ( $L$ is a configurable algorithm's parameter), which is used to compute an average test statistic value (line 3 in algorithm 2):

$$
\mathbb{T}_{i}^{\text {avg }}\left(\mathbf{T}_{i}\right)=\frac{1}{L} \sum_{l=1}^{L} \mathbb{T}_{i-L+l}\left(\mathbf{y}_{i-L+l}\right)
$$

where $\mathbb{T}_{i}^{\text {avg }}\left(\mathbf{T}_{i}\right)$ is the average test statistic value computed in the $i$-th sensing event based on the test statistic vector $\mathbf{T}_{i}=\left(\mathbb{T}_{i-L+1}\left(\mathbf{y}_{i-L+1}\right), \mathbb{T}_{i-L+2}\left(\mathbf{y}_{i-L+2}\right), \ldots, \mathbb{T}_{i-1}\left(\mathbf{y}_{i-1}\right), \mathbb{T}_{i}\left(\mathbf{y}_{i}\right)\right)$. In case that the test statistic $\mathbb{T}_{i}\left(\mathbf{y}_{i}\right)$ falls below the decision threshold $\lambda$, an additional check based on $\mathbb{T}_{i}^{\text {avg }}\left(\mathbf{T}_{i}\right)$ is then performed (line 7) before deciding the final channel state $S_{i}$. This additional check is aimed at preventing a busy channel from being declared to be idle as a result of an instantaneous signal energy drop (which depends on the particular signal energy variation pattern and radio propagation conditions) combined with a sufficiently short sensing period (which may be constrained by physical layer features or higher layer protocols). According to this additional verification, if the last 
sensing event reported an idle channel, $\mathbb{T}_{i}\left(\mathbf{y}_{i}\right)<\lambda$, but the average test statistic value (i.e., the average signal energy) of the last $L$ sensing events is greater than the decision threshold, $\mathbb{T}_{i}^{\operatorname{avg}}\left(\mathbf{T}_{i}\right)>\lambda$, this means that it is very likely that a signal is actually present in the sensed channel but the last sensing event resulted in $\mathbb{T}_{i}\left(\mathbf{y}_{i}\right)<\lambda$ due to an instantaneous energy drop of the received signal combined with a sufficiently short sensing period. As a result, the channel should be declared as busy (hypothesis $\mathcal{H}_{1}$ ) in such a case (line 8 ). On the other hand, if the last sensing event reported an idle channel, $\mathbb{T}_{i}\left(\mathbf{y}_{i}\right)<\lambda$, and the test statistic's average value of the last $L$ sensing events also indicates an idle channel, $\mathbb{T}_{i}^{\text {avg }}\left(\mathbf{T}_{i}\right)<\lambda$, this clearly means that the channel is actually idle. In such another case, hypothesis $\mathcal{H}_{0}$ can reliably be selected (line 10). With this formulation, the MED scheme aims at reducing the amount of misdetections caused by instantaneous signal energy drops, which would lead to an improved detection performance.

\section{IV.B. MED theoretical performance}

The test statistic values $\mathbb{T}_{i}\left(\mathbf{y}_{i}\right)$ can be assumed to be normally distributed (see equation 3 ) and mutually independent since they represent the energy of the sensed signal at time instants separated by time intervals much greater than the sensing period $N$ over which the signal energy is computed. Since $\mathbb{T}_{i}^{\text {avg }}\left(\mathbf{T}_{i}\right)$ is the average of independent and identically distributed (i.d.d.) Gaussian random variables, it also is normally distributed:

$$
\mathbb{T}_{i}^{\mathrm{avg}}\left(\mathbf{T}_{i}\right) \sim \mathcal{N}\left(\mu_{\mathrm{avg}}, \sigma_{\mathrm{avg}}^{2}\right)
$$

where $\mu_{\text {avg }}$ and $\sigma_{\text {avg }}^{2}$ are given by [19, eqs. 2.20 and 2.21]:

$$
\begin{aligned}
\mu_{\mathrm{avg}} & =\frac{M}{L} N\left(\sigma_{x}^{2}+\sigma_{w}^{2}\right)+\frac{L-M}{L} N \sigma_{w}^{2} \\
\sigma_{\mathrm{avg}}^{2} & =\frac{M}{L^{2}} 2 N\left(\sigma_{x}^{2}+\sigma_{w}^{2}\right)^{2}+\frac{L-M}{L^{2}} 2 N \sigma_{w}^{4}
\end{aligned}
$$

where $M \in[0, L]$ is the number of sensing events where a primary signal was actually present. Notice that the exact value of $M$ cannot be assumed to be known in practice. All that can be known is the output decisions $\mathcal{H}_{0} / \mathcal{H}_{1}$ of the spectrum sensing algorithm, which does not necessarily imply 
the presence/absence of a primary signal. This means that the performance of the MED algorithm cannot be predicted in practice since it depends on the particular channel occupancy pattern of the primary signal, which indeed is unknown. However, it can be lower- and upper-bounded by analyzing the extreme cases $M=0$ and $M=L$, which correspond to the case where the channel is always idle (for $M=0$ ) or always busy (for $M=L$ ) during the last $L$ sensing events.

Based on the distributions of $\mathbb{T}_{i}\left(\mathbf{y}_{i}\right)$ and $\mathbb{T}_{i}^{\text {avg }}\left(\mathbf{T}_{i}\right)$, the $P_{d}$ and $P_{f a}$ for the MED algorithm are:

$$
\begin{aligned}
P_{d}^{\mathrm{MED}} & =P\left\{\mathbb{T}_{i}\left(\mathbf{y}_{i}\right)>\lambda\right\}_{\mathcal{H}_{1}}+P\left\{\mathbb{T}_{i}\left(\mathbf{y}_{i}\right) \leq \lambda, \mathbb{T}_{i}^{\mathrm{avg}}\left(\mathbf{T}_{i}\right)>\lambda\right\}_{\mathcal{H}_{1}} \\
& =P\left\{\mathbb{T}_{i}\left(\mathbf{y}_{i}\right)>\lambda\right\}_{\mathcal{H}_{1}}+P\left\{\mathbb{T}_{i}\left(\mathbf{y}_{i}\right) \leq \lambda\right\}_{\mathcal{H}_{1}} \cdot P\left\{\mathbb{T}_{i}^{\mathrm{avg}}\left(\mathbf{T}_{i}\right)>\lambda \mid \mathbb{T}_{i}\left(\mathbf{y}_{i}\right) \leq \lambda\right\}_{\mathcal{H}_{1}}(12) \\
P_{f a}^{\mathrm{MED}} & =P\left\{\mathbb{T}_{i}\left(\mathbf{y}_{i}\right)>\lambda\right\}_{\mathcal{H}_{0}}+P\left\{\mathbb{T}_{i}\left(\mathbf{y}_{i}\right) \leq \lambda, \mathbb{T}_{i}^{\mathrm{avg}}\left(\mathbf{T}_{i}\right)>\lambda\right\}_{\mathcal{H}_{0}} \\
& =P\left\{\mathbb{T}_{i}\left(\mathbf{y}_{i}\right)>\lambda\right\}_{\mathcal{H}_{0}}+P\left\{\mathbb{T}_{i}\left(\mathbf{y}_{i}\right) \leq \lambda\right\}_{\mathcal{H}_{0}} \cdot P\left\{\mathbb{T}_{i}^{\mathrm{avg}}\left(\mathbf{T}_{i}\right)>\lambda \mid \mathbb{T}_{i}\left(\mathbf{y}_{i}\right) \leq \lambda\right\}_{\mathcal{H}_{0}}\left(1_{3}\right)
\end{aligned}
$$

Notice that $\mathbb{T}_{i}^{\text {avg }}\left(\mathbf{T}_{i}\right)$ and $\mathbb{T}_{i}\left(\mathbf{y}_{i}\right)$ are not completely independent since the computation of the former includes the value of the latter (see equation 8 ). However, $\mathbb{T}_{i}^{a v g}\left(\mathbf{T}_{i}\right)$ needs to be computed over a representative number of test statistic values in order to provide an acceptable estimate of the average signal energy in the sensed channel. Since the average of a relatively large set of values is not significantly affected, in general, by the particular value of a single element, it is therefore reasonable to assume for $L$ sufficiently large that $\mathbb{T}_{i}^{\text {avg }}\left(\mathbf{T}_{i}\right)$ can be considered to be approximately independent of $\mathbb{T}_{i}\left(\mathbf{y}_{i}\right)$, regardless of the actual channel state (busy or idle) in the $i$-th sensing event. Therefore, for $L$ sufficiently large:

$$
P\left\{\mathbb{T}_{i}^{\mathrm{avg}}\left(\mathbf{T}_{i}\right)>\lambda \mid \mathbb{T}_{i}\left(\mathbf{y}_{i}\right) \leq \lambda\right\}_{\mathcal{H}_{x}} \approx P\left\{\mathbb{T}_{i}^{\mathrm{avg}}\left(\mathbf{T}_{i}\right)>\lambda\right\}_{\mathcal{H}_{x}} \approx P\left(\mathbb{T}_{i}^{\mathrm{avg}}\left(\mathbf{T}_{i}\right)>\lambda\right)
$$

where $\mathcal{H}_{x}$ may be $\mathcal{H}_{0}$ or $\mathcal{H}_{1}$. Equations 12 and 13 then become:

$$
\begin{aligned}
P_{d}^{\mathrm{MED}} & \approx P_{d}^{\mathrm{CED}}+\left(1-P_{d}^{\mathrm{CED}}\right) \mathcal{Q}\left(\frac{\lambda-\mu_{\mathrm{avg}}}{\sigma_{\mathrm{avg}}}\right) \\
P_{f a}^{\mathrm{MED}} & \approx P_{f a}^{\mathrm{CED}}+\left(1-P_{f a}^{\mathrm{CED}}\right) \mathcal{Q}\left(\frac{\lambda-\mu_{\mathrm{avg}}}{\sigma_{\mathrm{avg}}}\right)
\end{aligned}
$$

The value of $\mathcal{Q}\left(\left(\lambda-\mu_{\mathrm{avg}}\right) / \sigma_{\mathrm{avg}}\right)$ in equations 15 and 16 depends on the particular channel occupancy pattern of the primary signal, which is unknown as already stated above. However, since 
$\mathcal{Q}(\cdot)$ is confined within the interval $[0,1]$, then $P_{d}^{\mathrm{MED}}$ and $P_{f a}^{\mathrm{MED}}$ are bounded by $P_{d}^{\mathrm{CED}} \leq P_{d}^{\mathrm{MED}} \leq$ 1 and $P_{f a}^{\mathrm{CED}} \leq P_{f a}^{\mathrm{MED}} \leq 1$. This means that the MED algorithm is able to improve the detection performance of the CED algorithm, which was its main motivation as discussed in Section IV.A.. However, such improvement is obtained at the expense of a false alarm probability degradation. To determine whether the MED scheme results in an overall performance improvement taking into account both $P_{d}$ and $P_{f a}$ with respect to the CED principle, Figure 2 depicts (based on equations 4, 5, 15 and 16) the ROC for both algorithms when $L=3, M \in[0, L], N=1000$, and the SNR values for which $P_{d}^{\mathrm{CED}}=0.9(-9.15 \mathrm{~dB}), P_{d}^{\mathrm{CED}}=0.8(-10.06 \mathrm{~dB}), P_{d}^{\mathrm{CED}}=0.7(-10.83$ $\mathrm{dB})$ and $P_{d}^{\mathrm{CED}}=0.6(-11.59 \mathrm{~dB})$ when $P_{f a}^{\mathrm{CED}}=0.1^{2}$. As appreciated, the MED performance depends on the primary signal's activity pattern (represented by means of $M$ ), but it is always inferior to that attained by the CED method.

The results of Figure 2 can be explained as follows. The MED formulation is able to reduce the amount of misdetections caused by instantaneous signal energy drops, which results in a $P_{d}$ improvement with respect to the CED algorithm as it has been inferred from equation 15. However, the resulting $P_{f a}$ increases to a greater extent thus leading to the degraded ROC observed in Figure 2. The $P_{f a}$ increase can be ascribed to the additional check performed in line 7 of the MED algorithm. When the primary signal ceases after some period of activity and the channel is released, there may be some following sensing events where $\mathbb{T}_{i}\left(\mathbf{y}_{i}\right)<\lambda$ due to the absence of the primary signal, but $\mathbb{T}_{i}^{\text {avg }}\left(\mathbf{T}_{i}\right)>\lambda$ due to the immediate past sensing events where the signal was still present. As a result, the additional check of line 7 may result in several consecutive false alarms. In the worst case, up to $L$ sensing events after the channel is released might result in false alarm

\footnotetext{
${ }^{2}$ Notice that CR networks are normally constrained by a maximum interference requirement, which can be mapped to a minimum detection probability that must be satisfied for SNR values above a predefined threshold, i.e. $P_{d}(\gamma) \geq$ $P_{d}^{\text {min }}$ for all $\gamma \geq \gamma_{\min }$. If we select $N=1000$ and $P_{f a}^{\mathrm{CED}}=0.1$, for a target $P_{d}^{\text {min }}=0.9$ the corresponding SNR is $\gamma_{\min }=-9.15 \mathrm{~dB}$ (equation 7). Therefore, this SNR value enables the evaluation of the potential improvements of the MED scheme with respect to the CED scheme in a realistic worst case. The SNR values for which $P_{d}^{\text {CED }}=0.8,0.7$ and 0.6 enable the evaluation of the MED performance under more unfavorable operating conditions.
} 
decisions, depending on the particular previous occupancy history and signal energy. In fact, as the number of previous sensing events where the primary signal was present, $M$, increases, the resulting $\mathbb{T}_{i}^{\text {avg }}\left(\mathbf{T}_{i}\right)$ becomes higher and the number of false alarms after the channel is released increases, as suggested by the ROC degradation observed in Figure 2 as $M$ increases. On the other hand, if the channel was sparsely used by the primary signal in the previous sensing events (low $M)$, the MED and CED behave similarly and their performances converge.

Based on the results of Figure 2, it can be concluded that the MED performance is upperbounded by the CED method. However, the previous analysis motivates the development of the improved method proposed in Section IV.C. and will make its understanding simpler.

\section{IV.C. IED operating principle}

Based on the analysis and discussion of Section IV.B., the IED scheme is proposed (see algorithm 3) in order to reduce the false alarm ratio of the MED scheme while preserving the detection performance improvement attained with respect to the CED method. The rationale of this proposal is illustrated in Figure 3. Two possible cases of interest are shown in sensing events number 15 (event A) and number 35 (event B). In both sensing events the MED scheme would select hypothesis $\mathcal{H}_{1}$ since $\mathbb{T}_{i}\left(\mathbf{y}_{i}\right)<\lambda$ and $\mathbb{T}_{i}^{\text {avg }}\left(\mathbf{T}_{i}\right)>\lambda$. While this decision would result in a detection performance improvement for sensing event $\mathrm{A}$, it would lead to a more significant false alarm degradation after sensing event number 30, where the channel is released, up to $L$ following sensing events (where event $\mathrm{B}$ is one of them). To avoid false alarms as those of event $\mathrm{B}$, the IED scheme performs an additional check (line 8 in algorithm 3) based on the test statistic of the previous sensing event, $\mathbb{T}_{i-1}\left(\mathbf{y}_{i-1}\right)$. When $\mathbb{T}_{i}\left(\mathbf{y}_{i}\right)<\lambda$ and $\mathbb{T}_{i}^{\text {avg }}\left(\mathbf{T}_{i}\right)>\lambda$, the condition $\mathbb{T}_{i-1}\left(\mathbf{y}_{i-1}\right)>\lambda$ indicates that $\mathbb{T}_{i}\left(\mathbf{y}_{i}\right)<\lambda$ may be due to an instantaneous energy drop, in which case hypothesis $\mathcal{H}_{1}$ should be selected (line 9). On the other hand, the condition $\mathbb{T}_{i-1}\left(\mathbf{y}_{i-1}\right)<\lambda$ suggests that $\mathbb{T}_{i}\left(\mathbf{y}_{i}\right)<\lambda$ may be due to the channel release, in which case hypothesis $\mathcal{H}_{0}$ should be selected (line 11). It is worth noting that the algorithm decisions could be based simply on $\mathbb{T}_{i}\left(\mathbf{y}_{i}\right)$ and 
$\mathbb{T}_{i-1}\left(\mathbf{y}_{i-1}\right)$. However, our experimental studies demonstrated that the additional use of $\mathbb{T}_{i}^{\text {avg }}\left(\mathbf{T}_{i}\right)$ can avoid misdetections for highly variable signals where various consecutive sensing events may be affected by instantaneous energy drops, in which case $\mathbb{T}_{i}\left(\mathbf{y}_{i}\right)<\lambda$ and $\mathbb{T}_{i-1}\left(\mathbf{y}_{i-1}\right)<\lambda$ even though a primary signal is actually present in the channel. Therefore, the MED and IED schemes would made the same decision in event A (hypothesis $\mathcal{H}_{1}$ ), thus improving the detection performance of the CED scheme. However, the IED scheme would select hypothesis $\mathcal{H}_{0}$ in event B, which would also decrease the false alarm ratio of the MED method. The IED proposal is therefore expected to improve the false alarm performance of the MED algorithm while still improving the detection performance of the CED method.

\section{IV.D. IED theoretical performance}

The test statistics $\mathbb{T}_{i}\left(\mathbf{y}_{i}\right), \mathbb{T}_{i-1}\left(\mathbf{y}_{i-1}\right)$ and $\mathbb{T}_{i}^{\text {avg }}\left(\mathbf{T}_{i}\right)$ can be assumed to be normally distributed as indicated by equations 3 and 9-11, respectively. Based on the distributions of $\mathbb{T}_{i}\left(\mathbf{y}_{i}\right), \mathbb{T}_{i-1}\left(\mathbf{y}_{i-1}\right)$ and $\mathbb{T}_{i}^{\text {avg }}\left(\mathbf{T}_{i}\right)$, the $P_{d}$ and $P_{f a}$ for the IED algorithm are:

$$
\begin{aligned}
P_{d}^{\mathrm{IED}} & =P\left\{\mathbb{T}_{i}\left(\mathbf{y}_{i}\right)>\lambda\right\}_{\mathcal{H}_{1}}+P\left\{\mathbb{T}_{i}\left(\mathbf{y}_{i}\right) \leq \lambda, \mathbb{T}_{i}^{\mathrm{avg}}\left(\mathbf{T}_{i}\right)>\lambda, \mathbb{T}_{i-1}\left(\mathbf{y}_{i-1}\right)>\lambda\right\}_{\mathcal{H}_{1}} \\
& =P\left\{\mathbb{T}_{i}\left(\mathbf{y}_{i}\right)>\lambda\right\}_{\mathcal{H}_{1}}+P\left\{\mathbb{T}_{i}\left(\mathbf{y}_{i}\right) \leq \lambda\right\}_{\mathcal{H}_{1}} \cdot P\left(\mathbb{T}_{i}^{\mathrm{avg}}\left(\mathbf{T}_{i}\right)>\lambda\right) \cdot P\left\{\mathbb{T}_{i-1}\left(\mathbf{y}_{i-1}\right)>\lambda\right\}_{\mathcal{H}_{1}} \\
P_{f a}^{\mathrm{IED}} & =P\left\{\mathbb{T}_{i}\left(\mathbf{y}_{i}\right)>\lambda\right\}_{\mathcal{H}_{0}}+P\left\{\mathbb{T}_{i}\left(\mathbf{y}_{i}\right) \leq \lambda, \mathbb{T}_{i}^{\mathrm{avg}}\left(\mathbf{T}_{i}\right)>\lambda, \mathbb{T}_{i-1}\left(\mathbf{y}_{i-1}\right)>\lambda\right\}_{\mathcal{H}_{0}} \\
& =P\left\{\mathbb{T}_{i}\left(\mathbf{y}_{i}\right)>\lambda\right\}_{\mathcal{H}_{0}}+P\left\{\mathbb{T}_{i}\left(\mathbf{y}_{i}\right) \leq \lambda\right\}_{\mathcal{H}_{0}} \cdot P\left(\mathbb{T}_{i}^{\mathrm{avg}}\left(\mathbf{T}_{i}\right)>\lambda\right) \cdot P\left\{\mathbb{T}_{i-1}\left(\mathbf{y}_{i-1}\right)>\lambda\right\}_{\mathcal{H}_{0}}
\end{aligned}
$$

where it has been assumed, following the same argument of Section IV.B., that $\mathbb{T}_{i}^{\text {avg }}\left(\mathbf{T}_{i}\right)$ can be considered to be approximately independent of both $\mathbb{T}_{i}\left(\mathbf{y}_{i}\right)$ and $\mathbb{T}_{i-1}\left(\mathbf{y}_{i-1}\right)$, regardless of the actual channel state (busy or idle) in the $i$-th and $(i-1)$-th sensing events, and that the test statistics of two consecutive sensing events, $\mathbb{T}_{i}\left(\mathbf{y}_{i}\right)$ and $\mathbb{T}_{i-1}\left(\mathbf{y}_{i-1}\right)$, can also be considered to be mutually 
independent. Based on such assumptions, equations 17 and 18 then become:

$$
\begin{aligned}
& P_{d}^{\mathrm{IED}} \approx P_{d}^{\mathrm{CED}}+P_{d}^{\mathrm{CED}}\left(1-P_{d}^{\mathrm{CED}}\right) \mathcal{Q}\left(\frac{\lambda-\mu_{\mathrm{avg}}}{\sigma_{\mathrm{avg}}}\right) \\
& P_{f a}^{\mathrm{IED}} \approx P_{f a}^{\mathrm{CED}}+P_{f a}^{\mathrm{CED}}\left(1-P_{f a}^{\mathrm{CED}}\right) \mathcal{Q}\left(\frac{\lambda-\mu_{\mathrm{avg}}}{\sigma_{\mathrm{avg}}}\right)
\end{aligned}
$$

Since $\mathcal{Q}(\cdot) \in[0,1], P_{d}^{\mathrm{IED}}$ and $P_{f a}^{\mathrm{IED}}$ are bounded by $P_{d}^{\mathrm{CED}} \leq P_{d}^{\mathrm{IED}} \leq 2 P_{d}^{\mathrm{CED}}-\left(P_{d}^{\mathrm{CED}}\right)^{2}$ and $P_{f a}^{\mathrm{CED}} \leq P_{f a}^{\mathrm{IED}} \leq 2 P_{f a}^{\mathrm{CED}}-\left(P_{f a}^{\mathrm{CED}}\right)^{2}$, meaning that the IED algorithm improves the detection performance of the CED algorithm at the expense of a false alarm probability degradation. Such degradation, however, is not as significant as in the case of the MED scheme, resulting in the improved ROC curve observed in Figure 4 for the IED algorithm (the figure has been obtained based on equations 4, 5, 19 and 20, and for the same operating conditions as Figure 2). As it can be appreciated, the IED performance is superior to that attained by the CED method. When the channel is idle in previous sensing events $(M=0)$, the IED scheme bases its decisions mostly on the test statistic of the current sensing event, $\mathbb{T}_{i}\left(\mathbf{y}_{i}\right)$, since for $M=0$ it is rather unlikely that $\mathbb{T}_{i}^{\text {avg }}\left(\mathbf{T}_{i}\right)>\lambda$. In this case the IED scheme behaves similar to the CED scheme and their performances agree. However, when the channel is busy in previous sensing events $(M>0)$, the IED scheme takes profit of this additional information to avoid misdetections due to instantaneous signal energy drops, as the MED scheme, but with a lower false alarm rate, which results in an overall improved performance. In fact, as $M$ increases, the IED method is able to avoid a higher amount of such misdetections. These results indicate that the IED performance is lower-bounded by the CED method: in the worst case IED performs as CED, but under favorable conditions it is able to attain significant performance improvements as highlighted by Figure 4.

\section{IV.E. IED threshold setting}

As discussed in Section III.C., the energy decision threshold is normally chosen to satisfy a certain target $P_{f a}$, which only requires the noise power to be known. In the particular case of the CED method, this is an straightforward problem that can be solved analytically based on equation 
5. The resulting decision threshold is given by equation 6 . In the case of the IED method, however, the procedure is not so straightforward because equation 20 cannot be solved for $\lambda$ analytically. In principle, the decision threshold required to meet a particular target $P_{f a}$ could be computed numerically based on equation 20. Nevertheless, this approach is a rather involved procedure from both computational and practical perspectives.

As an alternative, the following approach is proposed and analyzed. Let's assume that the decision threshold for the IED algorithm is selected according to the expression obtained for the CED scheme:

$$
\lambda=\left(\mathcal{Q}^{-1}\left(P_{f a, \text { target }}^{\text {IED }}\right) \sqrt{2 N}+N\right) \sigma_{w}^{2}
$$

where $P_{f a \text {,target }}^{\text {IED }}$ represents the target false alarm probability desired for the IED algorithm. Substituting equation 21 into equations 19 and 20 yields:

$$
\begin{aligned}
P_{d}^{\mathrm{IED}}(\gamma) & \approx P_{d}^{\mathrm{CED}}(\gamma)+P_{d}^{\mathrm{CED}}(\gamma)\left(1-P_{d}^{\mathrm{CED}}(\gamma)\right) \mathcal{Q}\left(\frac{\mathcal{Q}^{-1}\left(P_{f a, \text { target }}^{\mathrm{IED}}\right) \sqrt{2 N}-\frac{M}{L} N \gamma}{\sqrt{\frac{2 N}{L}\left\{1+\frac{M}{L}\left[(1+\gamma)^{2}-1\right]\right\}}}\right)(22) \\
& \approx P_{d}^{\mathrm{CED}}(\gamma)+P_{d}^{\mathrm{CED}}(\gamma)\left(1-P_{d}^{\mathrm{CED}}(\gamma)\right) \mathcal{Q}\left(\mathcal{Q}^{-1}\left(P_{f a, \text { target }}^{\mathrm{IED}}\right) \sqrt{L}-M \sqrt{\frac{N}{2 L}} \gamma\right)(23) \\
P_{f a}^{\mathrm{IED}}(\gamma) & \approx P_{f a, \text { target }}^{\mathrm{IED}}+P_{f a, \text { target }}^{\mathrm{IED}}\left(1-P_{f a, \text { target }}^{\mathrm{IED}}\right) \mathcal{Q}\left(\frac{\mathcal{Q}^{-1}\left(P_{f a, \text { target }}^{\mathrm{IED}}\right) \sqrt{2 N}-\frac{M}{L} N \gamma}{\sqrt{\frac{2 N}{L}\left\{1+\frac{M}{L}\left[(1+\gamma)^{2}-1\right]\right\}}}\right)(24) \\
& \approx P_{f a, \text { target }}^{\mathrm{IED}}+P_{f a, \text { target }}^{\mathrm{IED}}\left(1-P_{f a, \text { target }}^{\mathrm{IED}}\right) \mathcal{Q}\left(\mathcal{Q}^{-1}\left(P_{f a, \text { target }}^{\mathrm{IED}}\right) \sqrt{L}-M \sqrt{\frac{N}{2 L}} \gamma\right.
\end{aligned}
$$

where $P_{d}^{\mathrm{CED}}(\gamma)$ is given by equation 7 , replacing $P_{f a, \text { target }}^{\mathrm{CED}}$ with $P_{f a, \text { target }}^{\mathrm{IED}}$, and the second approximation of each equation assumes low SNR regime $(\gamma \ll 1)$. It is interesting to note, as opposed to the CED scheme, that the $P_{f a}$ for the IED method depends on the SNR and therefore on the received primary signal, which is explained by the fact that each sensing decision depends on the previous sensing results, where a signal might be present. Equation 22 indicates that the detection probability $P_{d}^{\mathrm{IED}}(\gamma)$ would improve with respect to the CED scheme, but the resulting $P_{f a}^{\mathrm{IED}}(\gamma)$ would be greater than $P_{f a \text {,target }}^{\mathrm{IED}}$ according to equation 24 . In the worst possible case, which cor- 
responds to $\mathcal{Q}(\cdot)=1$ in equation 24 , and assuming ${ }^{3}$ that $P_{f a, \text { target }}^{\mathrm{IED}} \ll 1$, the resulting false alarm rate would be $P_{f a}^{\mathrm{IED}}(\gamma) \approx 2 P_{f a \text {,target }}^{\mathrm{IED}}$. In order to guarantee that the resulting $P_{f a}^{\mathrm{IED}}(\gamma)$ does not exceed $P_{f a, \text { target }}^{\mathrm{IED}}$, even in the worst case, the required decision threshold must then be chosen as:

$$
\lambda=\left(\mathcal{Q}^{-1}\left(\frac{P_{f a, \text { target }}^{\mathrm{IED}}}{2}\right) \sqrt{2 N}+N\right) \sigma_{w}^{2}
$$

This alternative approach increases the decision threshold with respect to equation 21 and reduces the experienced $P_{f a}^{\mathrm{IED}}(\gamma)$, guaranteeing that the amount of lost spectrum opportunities does not exceed the ratio $P_{f a \text {,target }}^{\mathrm{IED}}$. Due to the existing trade-off between $P_{f a}$ and $P_{d}$, however, this also means that the resulting detection probability would be lower than that obtained when the decision threshold is simply selected according to equation 21 , in which case some potential interference might be caused to the primary system. Equation 26 can therefore be regarded as an aggressive threshold setting approach where the maximum amount of lost spectrum opportunities is strictly constrained at the expense of some potential risk of interference to the primary network. On the other hand, equation 21 can be regarded as a conservative approach because in this case a minimum detection performance is guaranteed but at the expense of sacrificing some spectrum opportunities since $P_{f a}^{\mathrm{IED}}(\gamma)>P_{f a, \text { target }}^{\mathrm{IED}}$.

The previous observations are illustrated and confirmed in Figure 5, where equations 22 and 24 (IED performance) are depicted for both conservative and aggressive approaches along with equation 7 (CED performance) for comparison purposes. The MED performance (equations 15 and 16) when the decision threshold is selected according to the conservative approach is also shown in order to corroborate the discussion of Section IV.B.. Although $P_{f a}^{\mathrm{IED}}(\gamma) \geq P_{f a \text {,target }}^{\mathrm{IED}}$ with the conservative approach, it is interesting to note that the resulting $P_{f a}$ degradation is appreciable when considering relatively large $P_{f a \text {,target }}^{\mathrm{IED}}$ values such as $P_{f a \text {,target }}^{\mathrm{IED}}=0.1$. For lower values (e.g., $\left.P_{f a, \text { target }}^{\mathrm{IED}}=0.01\right)$, the resulting $P_{f a}$ can be considered to be $P_{f a}^{\mathrm{IED}}(\gamma) \approx P_{f a, \text { target }}^{\mathrm{IED}}$ in practice. In such a case, the conservative approach is preferable over the aggressive alternative since it is

\footnotetext{
${ }^{3}$ For example, the IEEE 802.22 spectrum sensing requirements [16] specify that a CR terminal must be able to provide $P_{f a} \leq 0.1$. Lower values may be desirable as well in order to maximize spectrum utilization.
} 
able to provide the full $P_{d}$ improvements without a noticeable $P_{f a}$ degradation with respect to the CED scheme. The practical consequences of both threshold setting strategies are evaluated experimentally in Section IV.F..

\section{IV.F. IED experimental performance}

The experimental performance of the IED scheme in terms of $P_{d}$ and $P_{f a}$ is shown in Figure 6. These results have been obtained with the measurement platform and evaluation methodology described in [15]. The IED performance is shown as a function of the algorithm's parameter $L$. Notice that the values shown for $L=1$ correspond to the CED performance since the CED and IED schemes are equivalent for $L=1$. The performance has been obtained when the decision threshold is computed according to the conservative (equation 21) and aggressive (equation 26) approaches described in Section IV.E., and for various sensing periods $N$. For each sensing period, the SNR corresponding to $P_{d}^{\mathrm{CED}}=0.9$ and $P_{f a}^{\mathrm{CED}}=0.1$ is selected (according to equation $7, \mathrm{SNR}=4.29$ $\mathrm{dB}$ for $N=10, \mathrm{SNR}=-3.54 \mathrm{~dB}$ for $N=100$ and $\mathrm{SNR}=-9.15 \mathrm{~dB}$ for $N=1000)$. The target $P_{f a}$ for the IED algorithm is $P_{f a \text {,target }}^{\mathrm{IED}}=0.1$ in all cases.

As it can be appreciated, the IED proposal clearly outperforms the conventional CED scheme for all the considered sensing periods. As $L$ increases (i.e., the measured energies of more sensing events are combined when computing $\mathbb{T}_{i}^{\text {avg }}\left(\mathbf{T}_{i}\right)$ ), the true average signal energy can be estimated more accurately and the actual channel state can therefore be determined more reliably, which explains the growth of $P_{d}^{\text {IED }}$ as $L$ increases. However, for $L$ sufficiently large the true average signal energy can be estimated with reasonable accuracy. In such a case, further increasing $L$ does not significantly contribute to the estimation accuracy. As a result, increasing $L$ beyond certain value improves $P_{d}^{\text {IED }}$ marginally. Based on Figure $6, L=5$ can be considered as an appropriate trade-off between the obtained performance improvement and the amount of memory required to store the previous test statistic values for the sensed channels.

It is interesting to note, as expected from the discussion in Section IV.E., that the detection performance for the aggressive threshold setting approach is in general lower than that of the con- 
servative approach. This is indeed the price to be paid for the lower $P_{f a}$ attained by the aggressive approach, as shown in Figure 6. As it can be observed, the aggressive approach guarantees that $P_{f a, \text { target }}^{\mathrm{IED}}$ is never exceeded (which is not the case of the conservative one) at the expense of a lower detection probability and therefore an increased risk of potential interference to the primary network. Although $P_{f a}^{\mathrm{IED}} \geq P_{f a \text {,target }}^{\mathrm{IED}}$ for the conservative approach, as expected from equation 24, it can be observed however that in practice the resulting $P_{f a}^{\mathrm{IED}}$ can be made to be very similar to the desired target with the conservative approach if the appropriate $L$ is selected (e.g., the value $L=5$ mentioned above constitutes a reasonable choice). This indicates that the decision threshold of the IED algorithm can be selected following the same procedure as for the CED scheme (equation 21), which simplifies the design of the IED spectrum sensing algorithm.

Figure 7 compares the performance of the CED and IED (with $L=5$ ) algorithms in terms of the ROC. The results have been obtained by means of extensive simulations based on the same set of empirical data as Figure 6 and considering the same operating conditions. As it can be clearly appreciated, the IED algorithm is able to outperform the CED scheme for various radio technologies and sensing periods, providing important performance gains.

Based on the results of this section, it can be concluded that the IED approach is able to achieve significant detection performance improvements with respect to the CED method. It has been shown that the observed detection enhancements are not obtained at the expense of a noticeable false alarm degradation. Moreover, the algorithm's decision threshold can be selected according to the same analytical equation employed for the CED method, which simplifies the IED design and configuration procedure in a real CR system.

\section{COMPleXity ANALYSIS}

\section{V.A. Sample complexity}

The sample complexity of a spectrum sensing algorithm specifies how the sensing period $N$ required to meet a certain set of target $P_{d, \text { target }}^{\mathrm{IED}}$ and $P_{f a, \text { target }}^{\mathrm{IED}}$ scales as the experienced SNR $\gamma$ 
degrades. For the CED method, the sample complexity can be determined by solving equation 7 for $N$, which yields:

$$
N=2\left[\frac{Q^{-1}\left(P_{f a, \text { target }}^{\mathrm{IED}}\right)-Q^{-1}\left(P_{d, \text { target }}^{\mathrm{IED}}\right)(1+\gamma)}{\gamma}\right]^{2}
$$

Assuming low SNR regime $(\gamma \ll 1)$, the required number of samples scales as $O\left(1 / \mathrm{SNR}^{2}\right)$, which represents the sample complexity of the CED algorithm.

For the IED method, the sample complexity cannot be determined analytically from equations 22 and 24. However, equations 19 and 20 constitute a system of two equations that can be solved numerically as a function of $N$ and $\lambda$ for various SNR values. The obtained $N$ versus the considered SNR is depicted in Figure 8, which enables the comparison of the sample complexity for the CED and IED approaches. The curves corresponding to the IED method are a shifted version of the CED curve, meaning that the IED sample complexity is also given by $O\left(1 / \mathrm{SNR}^{2}\right)$.

\section{V.B. Computational complexity}

The computational cost of the CED and IED methods can be estimated by comparing the set of calculations to be performed in algorithms 1 and 3 . First, the computation of $\mathbb{T}_{i}\left(\mathbf{y}_{i}\right)$ requires $N$ products and $N-1$ sums, which is common to both algorithms. Additionally, the IED algorithm computes $\mathbb{T}_{i}^{\text {avg }}\left(\mathbf{T}_{i}\right)$, which requires $L-1$ sums and one division, performs two additional comparisons (lines 7 and 8 in algorithm 3), and requires memory to store the last $L-1$ test statistic values for each channel the $\mathrm{CR}$ is sensing and operating over. The computational cost increase of the IED method can therefore be considered to be negligible, especially when compared to other more sophisticated methods such as cyclostationary feature detectors $[10,11]$ or covariance-based detectors [12], which require more computationally complex operations such as sample autocorrelation and covariance matrices.

To illustrate the computational costs of the CED and IED schemes, Figure 8 compares the average computation times observed in the simulations performed in the context of this work, based on Matlab running in a PC with an Intel Core2 Quad processor at $2.4 \mathrm{GHz}$. As it can be appreciated, 
the IED computation time is approximately the same for $L=1$ and $L=100$, meaning that the computation of the average test statistic in line 3 of algorithm 3 is negligible and that most of the additional time with respect to the CED scheme is due to the additional verifications performed in lines 7 and 8 of algorithm 3. The time required to perform these verifications, however, becomes less significant with respect to the time required to compute $\mathbb{T}_{i}\left(\mathbf{y}_{i}\right)$ as the sensing period $N$ increases. As a result, the computation time of the CED and IED algorithms is approximately the same for the sensing periods normally required in practice (see Figure 1). Despite the implemented code was not optimized and a general purpose PC was employed, these results are enough to confirm that the computational cost increase of the IED algorithm is negligible in practice.

\section{Discussion}

The IED spectrum sensing method proposed and evaluated in this work exploits the history of past spectrum sensing results to avoid signal misdetections caused by instantaneous energy drops, which may be due to the radio channel fading properties or the primary transmission power pattern. The use of past spectrum sensing results to infer the current channel state is sensible if the sensing events are reasonably close in time. This means that the full detection performance improvement of the IED scheme would be achieved when the target channel is sensed with enough periodicity. However, if the sensing frequency is increased while the sensing interval $N$ is kept constant, the time devoted to sensing increases and therefore the time available for data transmission decreases, which results in a throughput degradation for the secondary system [20]. To maintain the same average throughput, the sensing interval $N$ should be decreased along with the period between two consecutive sensing events. However, if the sensing period $N$ is made sufficiently short, the test statistic values may follow the instantaneous variations of the received signal energy. This might result in some signal misdetections in the particular case of the CED method as discussed in Section III.D., which is avoided with the IED method if the spectrum sensing operation is configured properly. Therefore, the IED improvements would be obtained with high sensing frequencies 
and short sensing intervals. Notice that shortening the sensing interval means that the detection probability in each isolated sensing event decreases, but if several consecutive sensing events that are sufficiently close in time are combined according to the IED principle, the same detection performance would be achieved as the obtained results indicate. In such a case, the benefit obtained would be an enhanced spectrum agility as a result of the increased sensing frequency ${ }^{4}$. Therefore, a proper configuration of the IED method would be able to improve the detection performance of the CED method or preserve the same performance while increasing the spectrum agility, thus enabling CRs to exploit spectrum gaps sooner and take profit of shorter spectrum opportunities. The ultimate consequence would be an improvement of the overall spectrum usage efficiency.

\section{Conclusions}

Energy detection has gained popularity as a spectrum sensing technique for cognitive radio due to its simplicity and general applicability (regardless of the signal format to be detected) as well as its low computational and implementation costs. Its main drawback, however, is its well-known detection performance limitations. Various alternative spectrum sensing methods have been shown to outperform energy detection, but at the expense of significantly increased computational cost and limited field of applicability since such methods have usually been devised for the improved detection of particular signal formats. This work has proposed an improved energy detection method that is able to outperform the classical energy detection scheme while preserving a similar level of complexity and computational cost as well as its general field of applicability. The algorithm performance has been assessed analytically and corroborated with experimental results, demonstrating the capabilities of the proposed approach.

\footnotetext{
${ }^{4}$ As a numerical example, and according to Figure 8 , the number of samples required for $\mathrm{SNR}=-10 \mathrm{~dB}$, $P_{d, \text { target }}=0.9$ and $P_{f a, \text { target }}=0.1$ would be $N^{C E D} \approx 1500$ and $N^{I E D} \approx 1000$ for the CED and IED methods, respectively. If the transmit-to-sensing time ratio is denoted as $\eta$, the time between two consecutive sensing events would be given by $N^{C E D}(1+\eta)$ and $N^{I E D}(1+\eta)$, which is 1.5 times $(33 \%)$ lower in the case of the IED method for the same $P_{d \text {,target }}, P_{f a, \text { target }}$ and $\eta$.
} 


\section{ACKNOWLEDGements}

The authors wish to acknowledge the activity of the Network of Excellence in Wireless COMmunications NEWCOM++ of the European Commission (contract n. 216715) that motivated this work. The support from the Spanish Ministry of Science and Innovation (MICINN) under FPU grant AP2006-848 is hereby acknowledged.

\section{REFERENCES}

[1] S. Haykin, "Cognitive radio: brain-empowered wireless communications," IEEE Journal on Selected Areas in Communications, vol. 23, no. 2, pp. 201-220, Feb. 2005.

[2] I. F. Akyildiz, W.-Y. Lee, M. C. Vuran, and S. Mohanty, "A survey on spectrum management in cognitive radio networks," IEEE Communications Magazine, vol. 46, no. 4, pp. 40-48, Apr. 2008.

[3] A. Ghasemi and E. S. Sousa, "Spectrum sensing in cognitive radio networks: requirements, challenges and design trade-offs," IEEE Communications Magazine, vol. 46, no. 4, pp. 32-39, Apr. 2008.

[4] T. Yücek and H. Arslan, "A survey of spectrum sensing algorithms for cognitive radio applications," IEEE Communications Surveys and Tutorials, vol. 11, no. 1, pp. 116-130, First Quarter 2009.

[5] D. D. Ariananda, M. K. Lakshmanan, and H. Nikookar, "A survey on spectrum sensing techniques for cognitive radio," in Proceedings of the Second International Workshop on Cognitive Radio and Advanced Spectrum Management (CogART 2009), May 2009, pp. 74-79.

[6] D. Noguet et al., "Sensing techniques for cognitive radio - state of the art and trends," Oct. 2009, IEEE SCC 41 P1900.6 White paper, available at http://grouper.ieee.org/groups/scc41/6/documents/ white_papers/P1900.6_WhitePaper_Sensing_final.pdf.

[7] H. Urkowitz, "Energy detection of unknown deterministic signals," Proceedings of the IEEE, vol. 55, no. 4, pp. 523-531, Apr. 1967.

[8] R. Price and N. Abramson, "Detection theory," IEEE Transactions on Information Theory, vol. 7, no. 3, pp. 135-139, Jul. 1961.

[9] J. G. Proakis, Digital communications, 5th ed. McGraw-Hill, 2008.

[10] W. A. Gardner, "Signal interception: a unifying theoretical framework for feature detection," IEEE Transactions on Communications, vol. 36, no. 8, pp. 897-906, Aug. 1988.

[11] W. A. Gardner and C. M. Spooner, "Signal interception: performance advantages of cyclic-feature detectors," IEEE Transactions on Communications, vol. 40, no. 1, pp. 149-159, Jan. 1992.

[12] Y. Zeng and Y.-C. Liang, "Spectrum-sensing algorithms for cognitive radio based on statistical covariances," IEEE Transactions on Vehicular Technology, vol. 58, no. 4, pp. 1804-1815, May 2009.

[13] D. J. Thomson, "Spectrum estimation and harmonic analysis," Proceedings of the IEEE, vol. 70, no. 9, pp. 1055-1096, Sep. 1982.

[14] B. Farhang-Boroujeny, "Filter bank spectrum sensing for cognitive radios," IEEE Transactions on Signal Processing, vol. 56, no. 5, pp. 1801-1811, May 2008.

[15] M. López-Benítez, F. Casadevall, and C. Martella, "Performance of spectrum sensing for cognitive radio based on field measurements of various radio technologies," in Proceedings of the 16th European Wireless Conference (EW 2010), Special session on Cognitive Radio, Apr. 2010, pp. 1-9.

[16] S. Shellhammer and G. Chouinard, "Spectrum sensing requirements summary," Jul. 2006, IEEE 802.2206/0089r5.

[17] M. Abramowitz and I. A. Stegun, Handbook of mathematical functions with formulas, graphs, and mathematical tables, 10th ed. New York: Dover, 1972.

[18] J. J. Lehtomaki, M. Juntti, H. Saarnisaari, and S. Koivu, "Threshold setting strategies for a quantized total power radiometer," IEEE Signal Processing Letters, vol. 12, no. 11, pp. 796-799, Nov. 2005.

[19] D. Blumenfeld, Operations research calculations handbook. C CRC Press, 2001.

[20] Y.-C. Liang, Y. Zeng, E. C. Y. Peh, and A. T. Hoang, "Sensing-throughput tradeoff for cognitive radio networks," IEEE Transactions on Wireless Communications, vol. 7, no. 4, pp. 1326-1337, Apr. 2008. 


\section{ALGORITHMS}




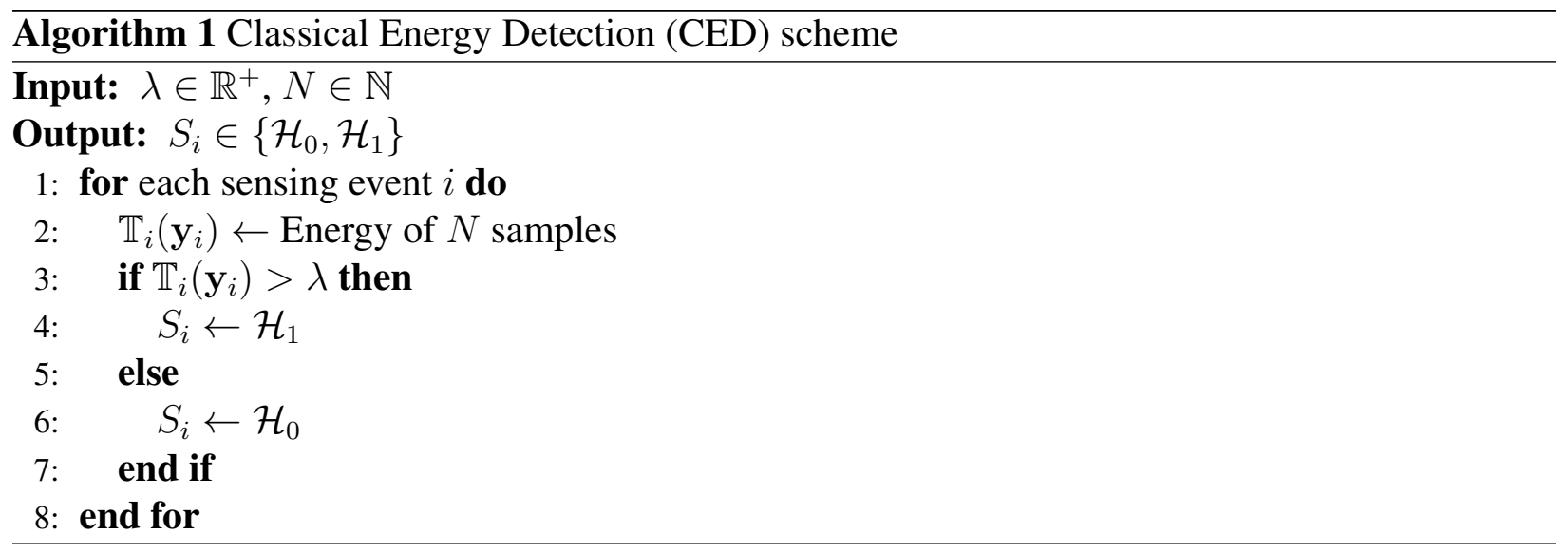




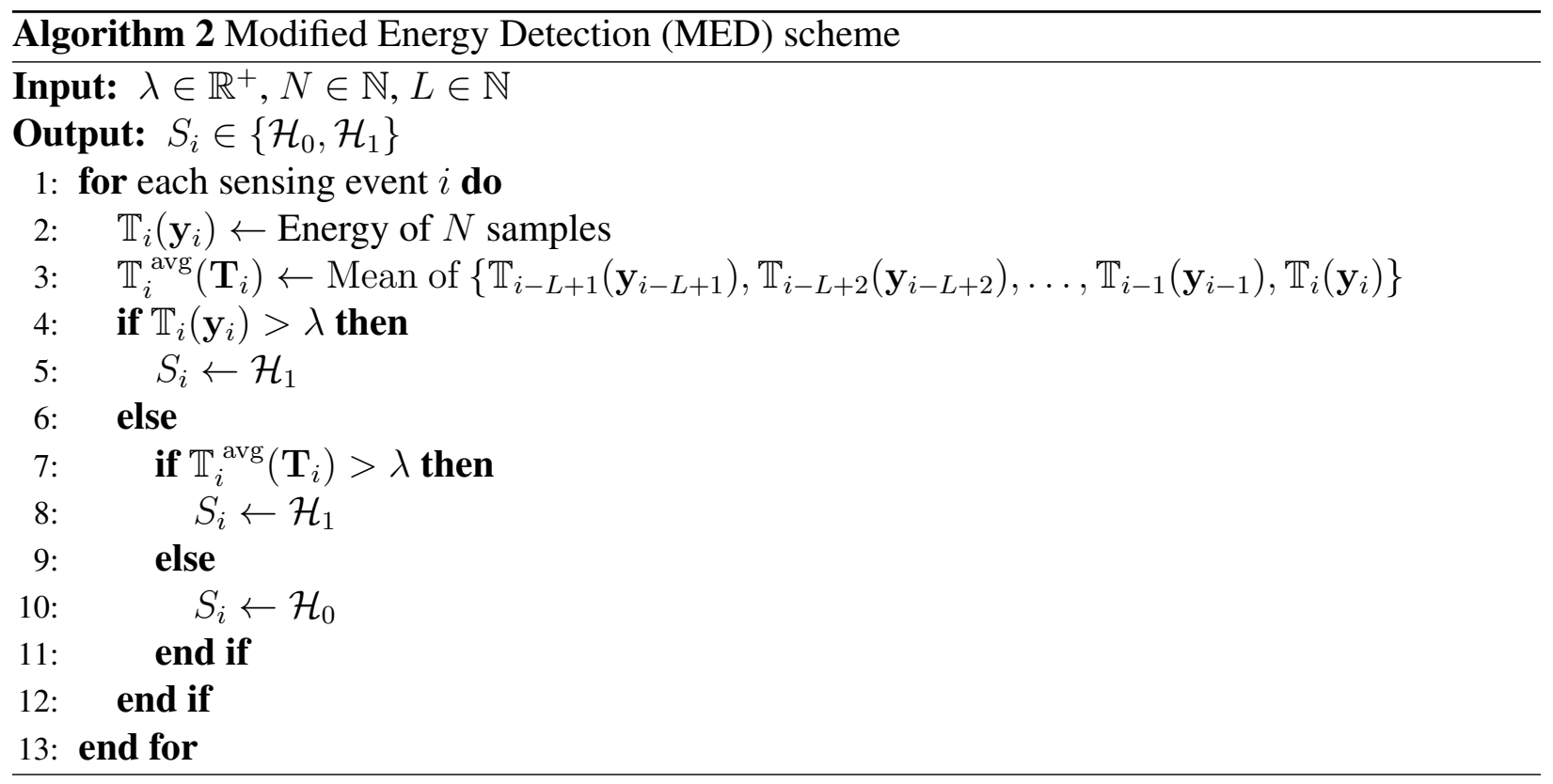




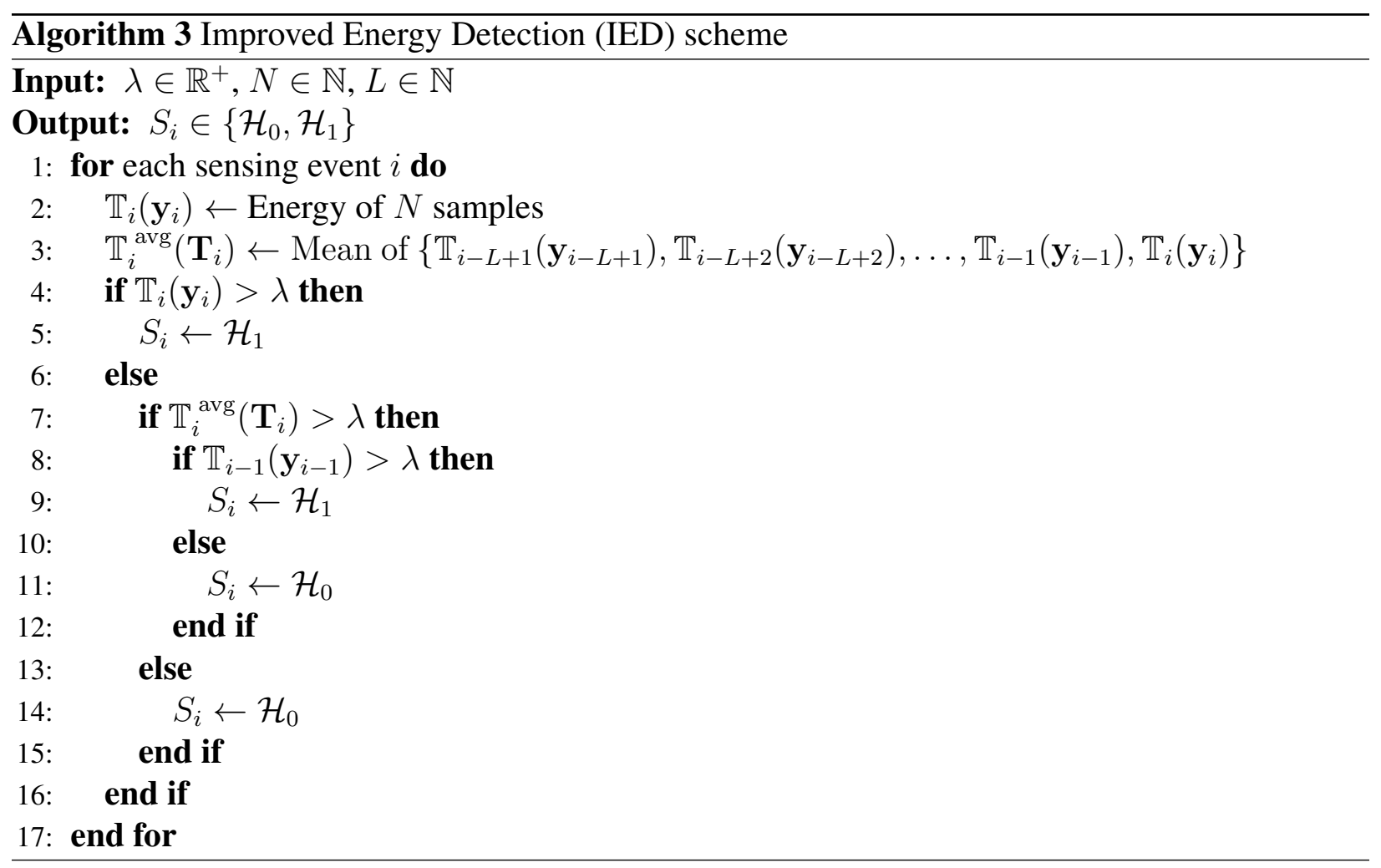




\section{FIGURES}




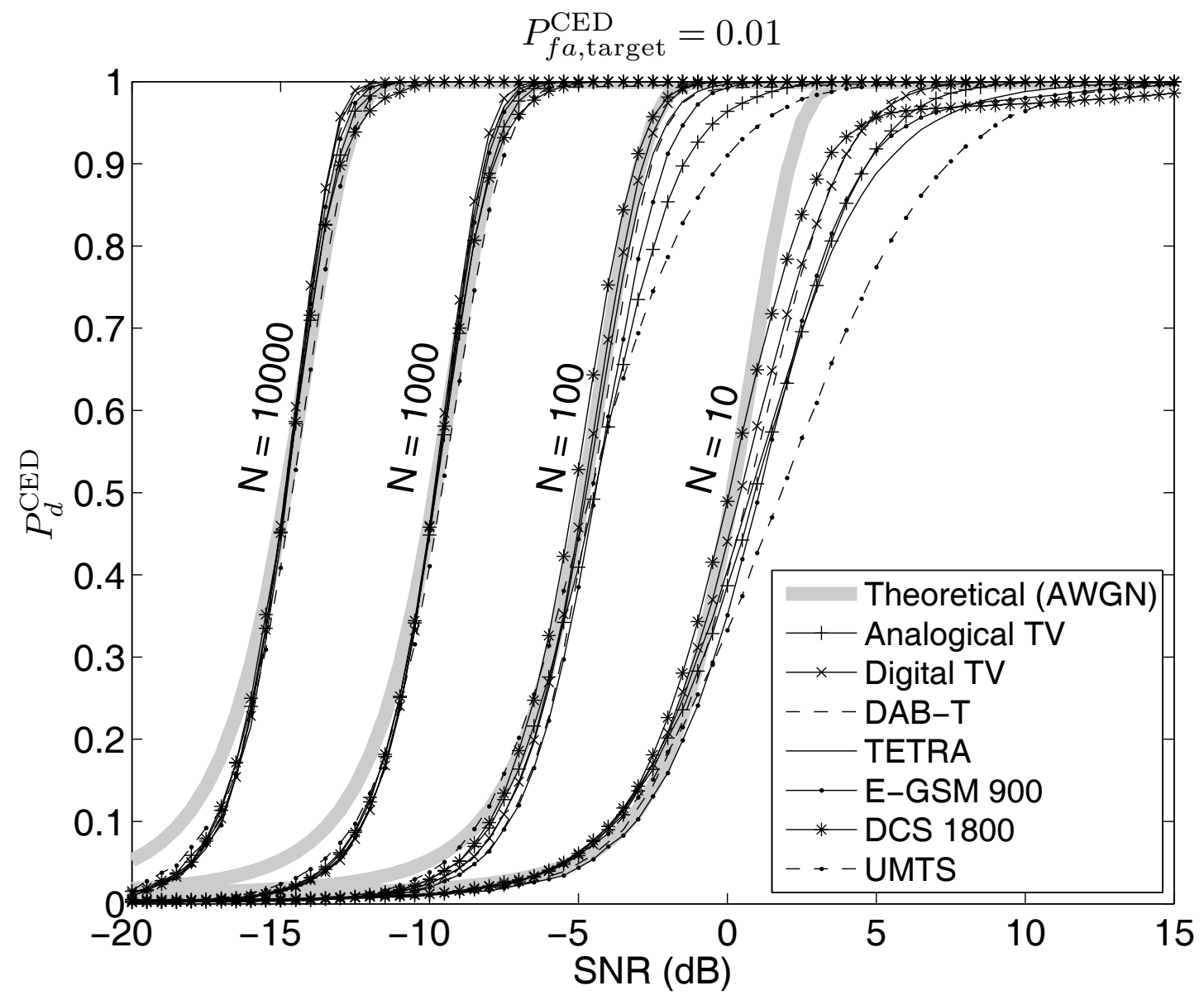

Figure 1: Theoretical and experimental performance for the CED algorithm. 

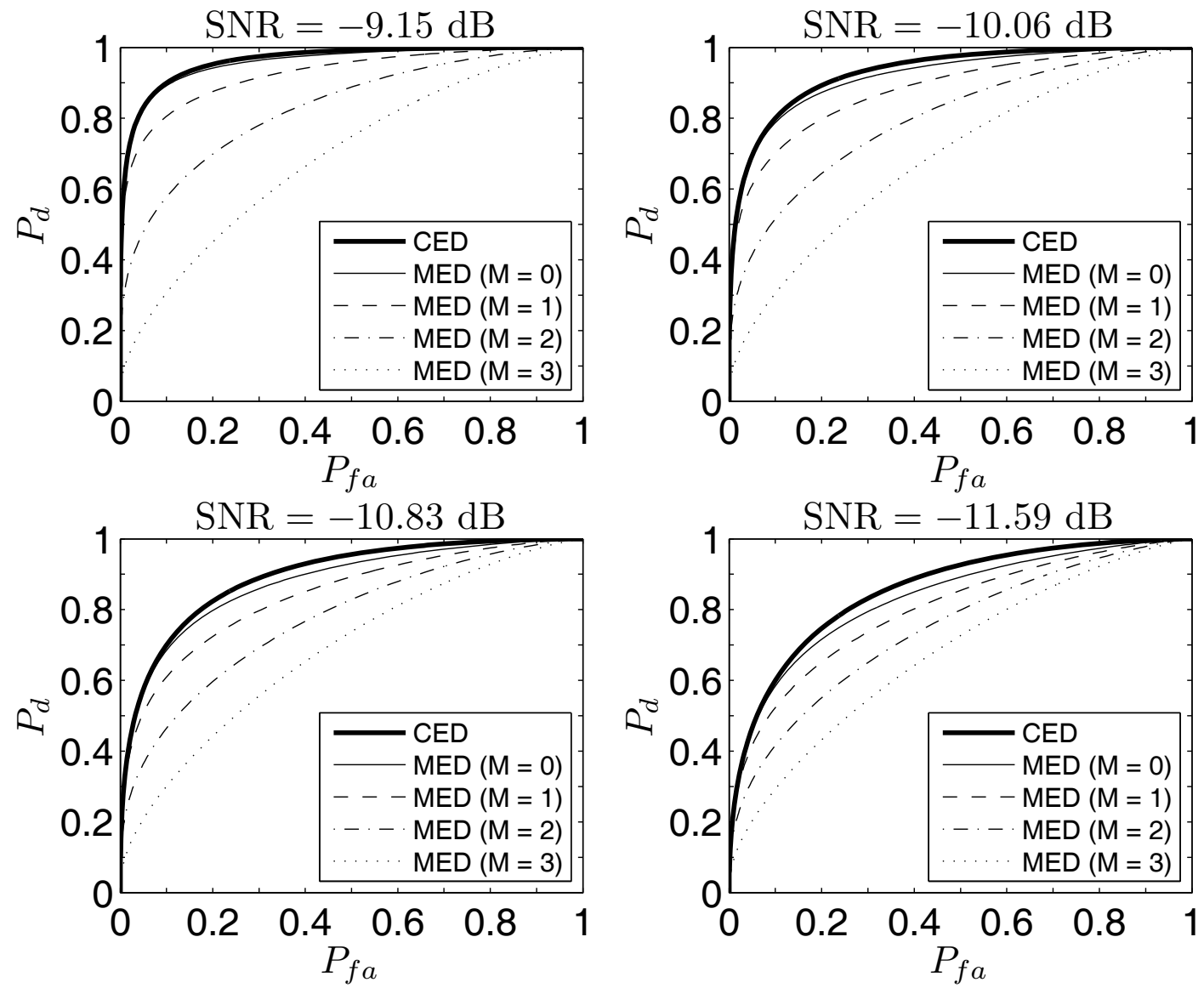

Figure 2: ROC curve for the CED and MED algorithms ( $L=3, M \in[0, L], N=1000)$. 


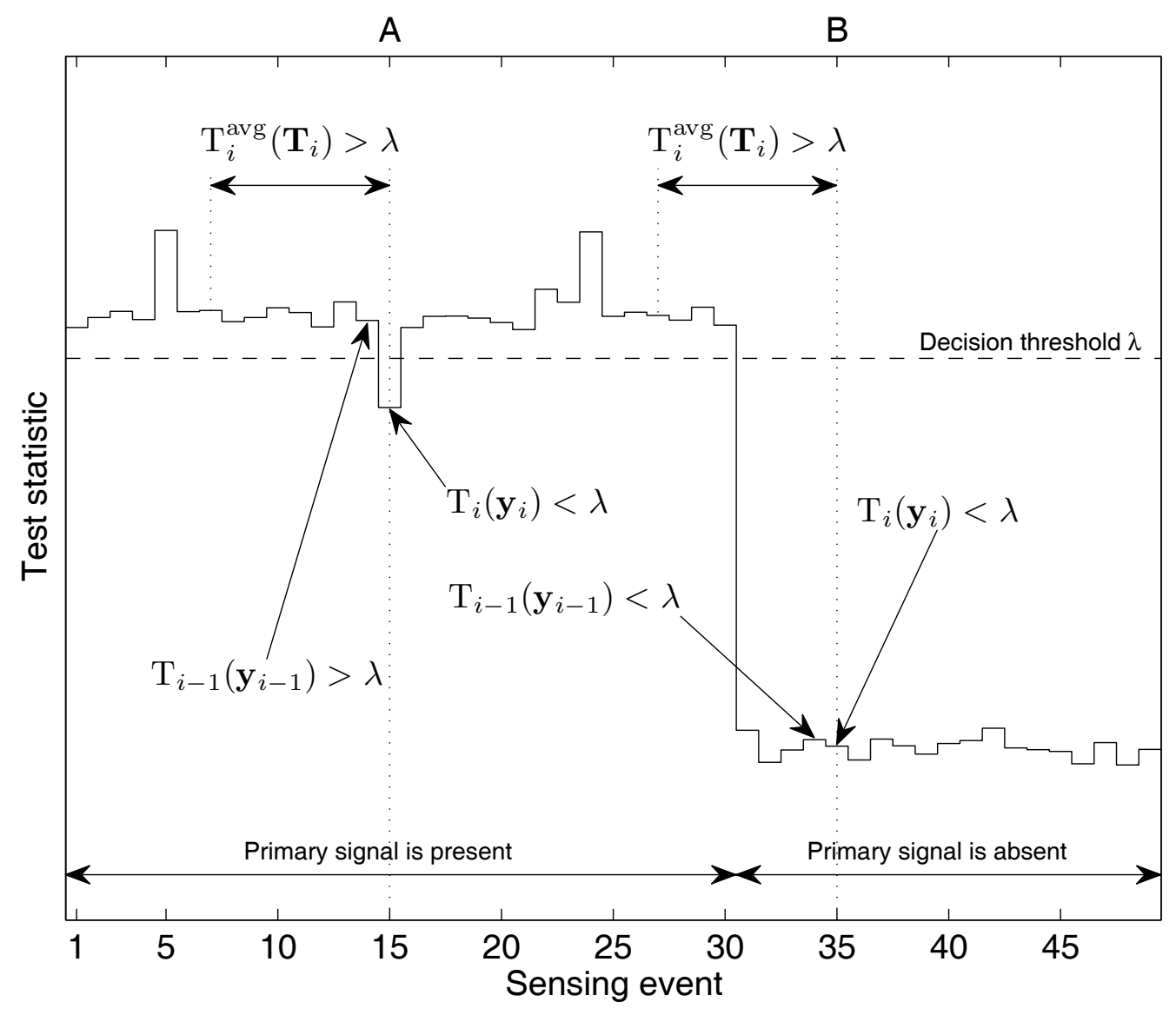

Figure 3: Rationale for the IED proposal. 

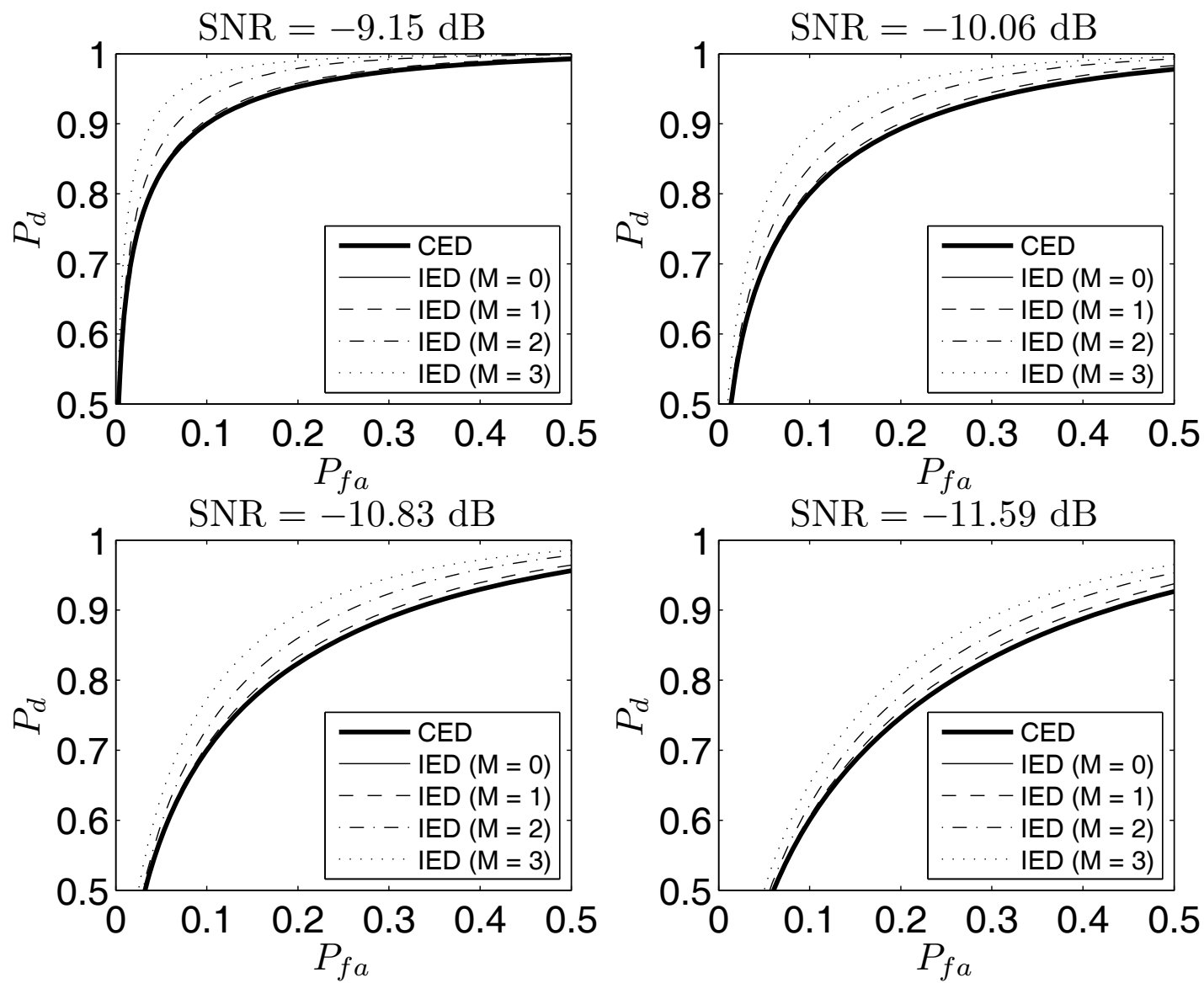

Figure 4: ROC curve for the CED and IED algorithms ( $L=3, M \in[0, L], N=1000)$. 


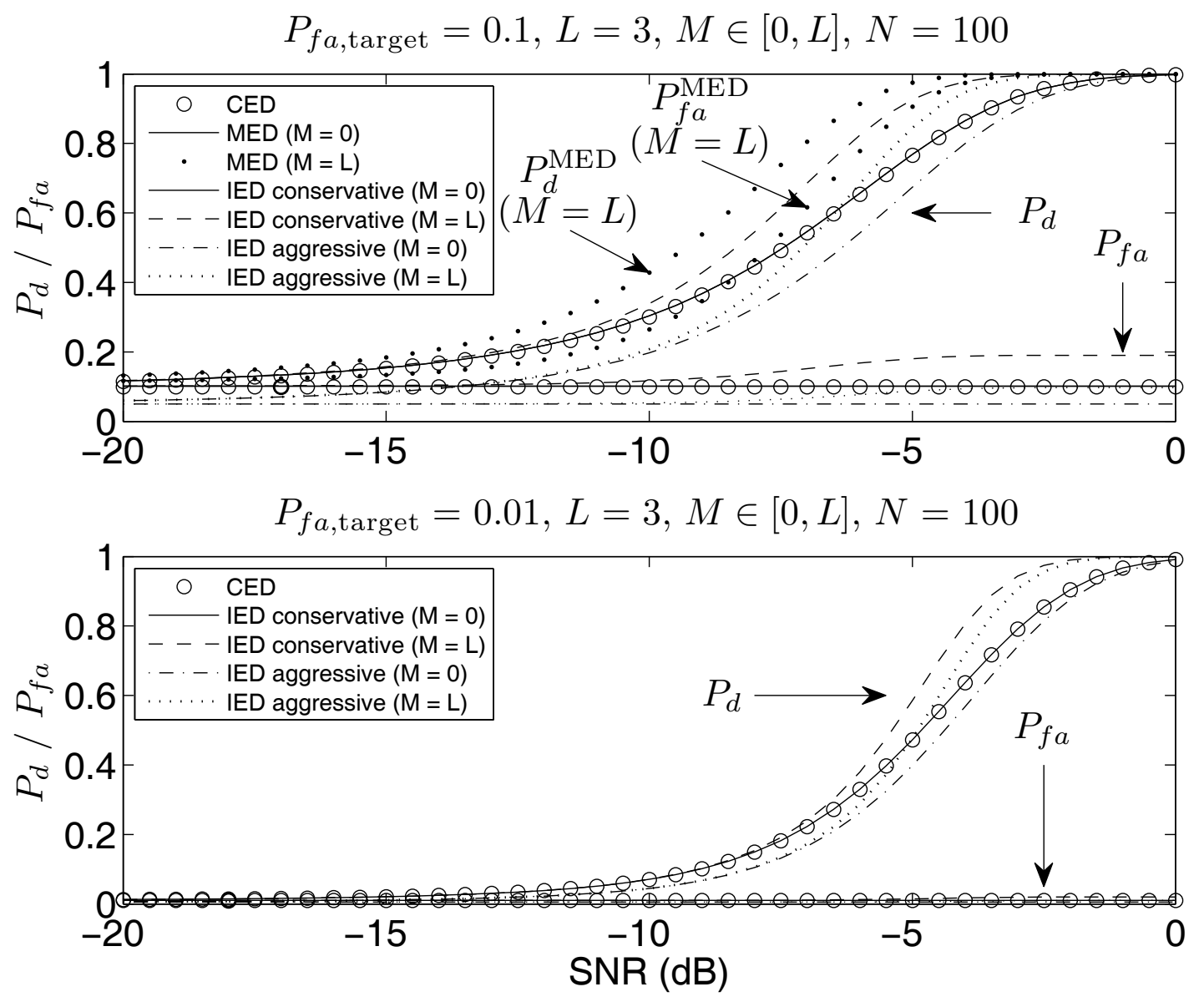

Figure 5: Theoretical performance for the CED, MED and IED algorithms. 


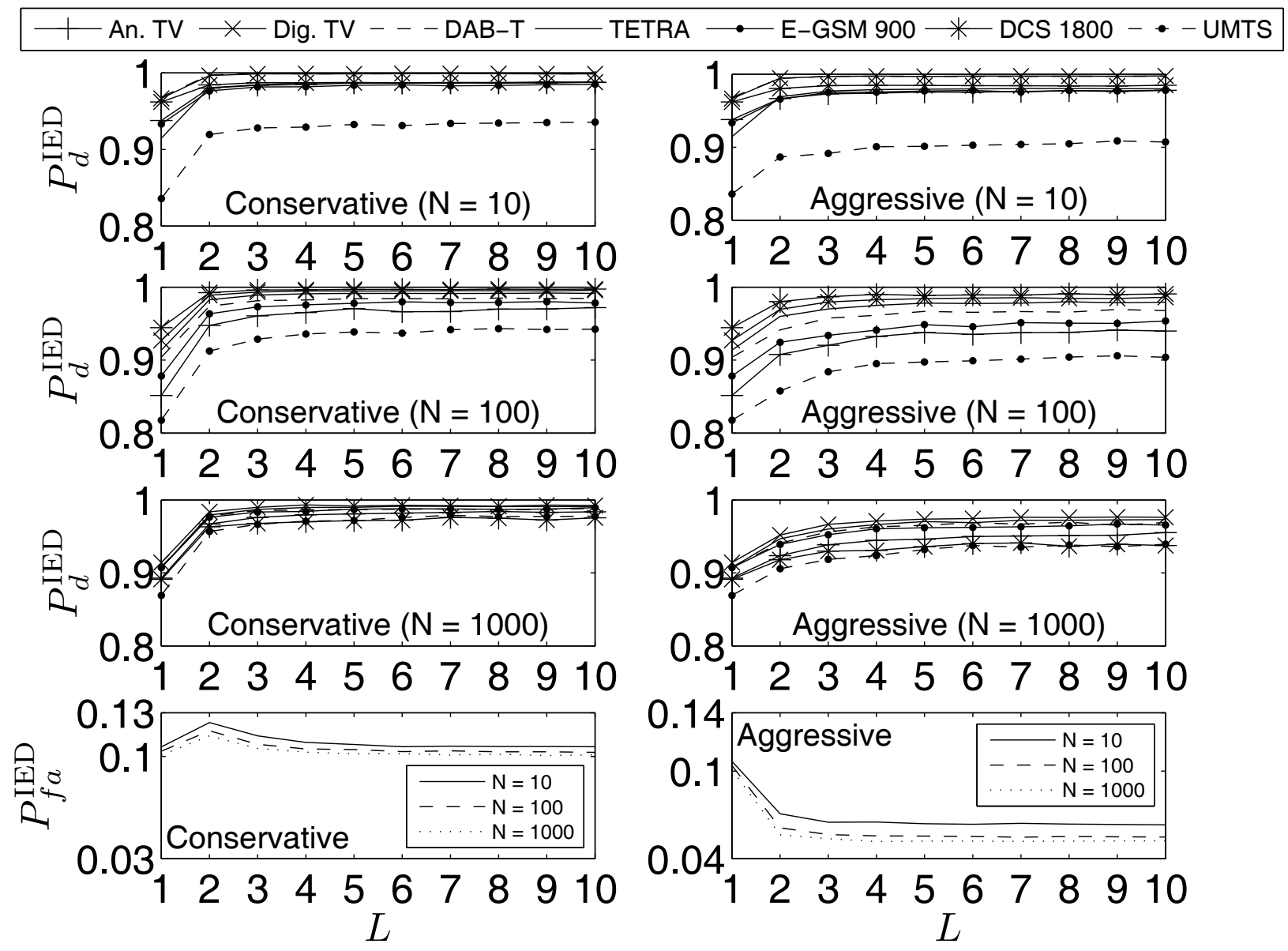

Figure 6: Experimental detection and false alarm performance for the IED algorithm. 

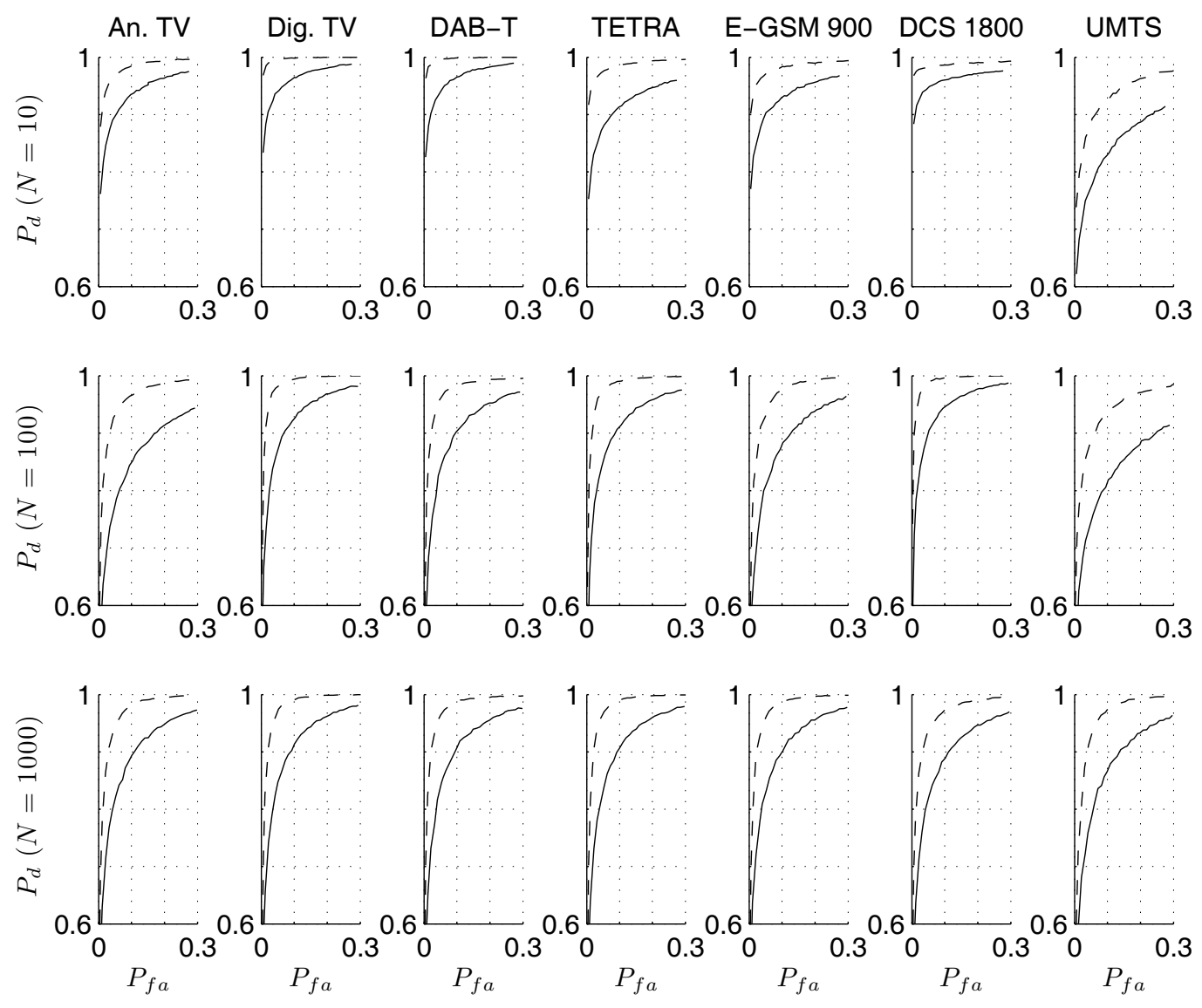

Figure 7: Experimental ROC curve for the CED (solid line) and IED (dashed line) algorithms. 

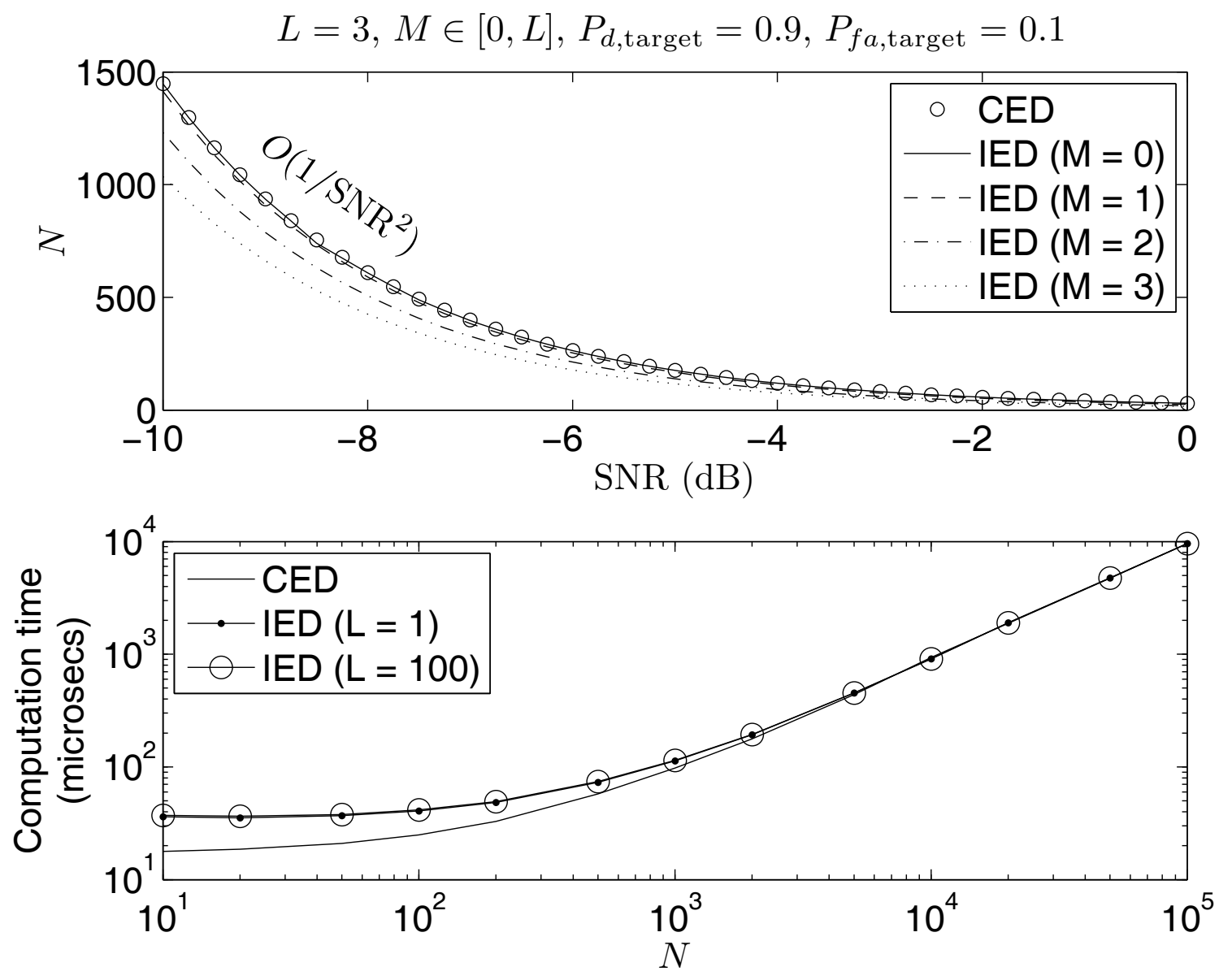

Figure 8: Complexities of the CED and IED algorithms (upper graph: sample complexity; lower graph: computational complexity). 